\title{
Control Effectiveness Analysis of the hawkmoth Manduca sexta: a Mul- tibody Dynamics Approach
}

\author{
Joong-Kwan Kim* and Jae-Hung Han** \\ Department of Aerospace Engineering, Korea Advanced Institute of Science and Technology, 291 Daehak-ro, Yuseong-gu, \\ Daejeon, Republic of Korea.
}

\begin{abstract}
This paper presents a control effectiveness analysis of the hawkmoth Manduca sexta. A multibody dynamic model of the insect that considers the time-varying inertia of two flapping wings is established, based on measurement data from the real hawkmoth. A six-degree-of-freedom (6-DOF) multibody flight dynamics simulation environment is used to analyze the effectiveness of the control variables defined in a wing kinematics function. The aerodynamics from complex wing flapping motions is estimated by a blade element approach, including translational and rotational force coefficients derived from relevant experimental studies. Control characteristics of flight dynamics with respect to the changes of three angular degrees of freedom (stroke positional, feathering, and deviation angle) of the wing kinematics are investigated. Results show that the symmetric (asymmetric) wing kinematics change of each wing only affects the longitudinal (lateral) flight forces and moments, which implies that the longitudinal and lateral flight controls are decoupled. However, there are coupling effects within each plane of motion. In the longitudinal plane, pitch and forward/backward motion controls are coupled; in the lateral plane, roll and side-translation motion controls are coupled.
\end{abstract}

Key words: hawkmoth Manduca sexta, control effectiveness, flight dynamics, insect-inspired, flapping-wing MAVs, multibody dynamics

\section{Introduction}

Insects perform highly maneuverable yet stable flight that is rarely found from manmade fixed- or rotary-wing aircraft. It is also remarkable that they manage all these maneuvers through only a pair of wings, whereas manmade aircraft utilize thrusters and various other control surfaces, such as flaps, rudders, and ailerons, to maintain their flight. The wings of the insect can be defined to have three independent rotational degrees of freedom (stroke positional, feathering, and deviation angle) [1], and it is believed that the insect can control each of them, with their complex muscles and exoskeleton structure. However, when it comes to an engineered implementation in an insect-inspired micro air vehicle (MAV), there are extreme difficulties, because of the very tight weight budget of the system. At present, only a limited number of actuators and limited complexity of mechanisms are possible to be installed in a flyable flapping wing micro air vehicle (MAV) [2], thus the control characteristics of the system can easily be underactuated. Therefore, the key thing for implementing flight control to the flapping MAV is how to obtain full control authorities to all of the six degrees of freedom motions, with a limited number of possible control inputs.

To better understand the fundamental mechanics of the flight control of the insect, and to utilize the knowledge for realizing manmade flapping MAVs, several interdisciplinary studies between biology and aerospace engineering have been conducted. High-speed footage recording/processing techniques for the stimulated (disturbed) flying insect $[3,4]$ are used to investigate bio-inspired control strategies of the insects: which sensory organ it uses, which state it feeds back, which muscle it activates, and consequently, which control strategy it uses to evade flight instability. However, this
This is an Open Access article distributed under the terms of the Creative Commons Attribution Non-Commercial License (http://creativecommons.org/licenses/by$\mathrm{nc} / 3.0 /$ which permits unrestricted non-commercial use, distribution, and reproduction in any medium, provided the original work is properly cited.

\footnotetext{
(c) * Graduate student, Dept of Aerospace Engineering

** Professor, Corresponding author: jaehunghan@kaist.ac.kr
} 
inference process has a limitation, in that it can only provide us with input/output sets and inferred control strategy based on hypothesis testing. A simplified mathematical modeling of the insect flight dynamics and hardware development/test has also been conducted [5-8]. Through this mathematical modeling approach, more direct clues can be provided, such as a quantitative relation between given control inputs, and the consequent dynamic responses. However, several assumptions for a simplified modeling make it hard to capture the time-varying nonlinear characteristics of the insect flight dynamics. Frequently used assumptions are cycle-averaged aerodynamics, simple single rigid body equations of motion that ignore the inertia effects of flapping wings, and linearization of the equations of motion.

In this study, an at-scale multibody dynamic model of the hawkmoth Manduca sexta is established, to consider time-varying inertia of the flapping wings. The timevarying inertia of the flapping wings is important, because of the fast flapping frequency of the insect, ranging from a few tens to hundreds of $\mathrm{Hz}$. In this dynamic range, even though the wings have relatively smaller mass than the body, inertial forces of the wings play a role as important as the aerodynamic forces [9]. In particular, asymmetric wing kinematics induces more unbalanced inertial forces. Thus the wing's time-varying inertial change needs to be taken into account, when investigating the force and moment changes under a given wing kinematics command variation. Based on this multibody insect model, control effectiveness analysis is performed using a modified version of the multibody dynamics simulation environment, developed in the author's previous studies $[10,11]$. Changes in six degrees of freedom forces and moments with respect to the variation of the three angular degrees of freedom wing kinematics are investigated, and their characteristics are analyzed.

\section{Material and Methods}

\subsection{Insect model and coordinate systems}

The hawkmoth Manduca sexta is a relatively large flying insect, capable of hovering flight with a flapping frequency of about $26 \mathrm{~Hz}$ [21]. It has two sets of wings, and each set consists of fore- and hind-wing, which flap in a synchronized manner, as if they were connected. Also, the flight is being driven primarily by the motion of the fore-wings only [12]. For this study, the fore- and hind-wings are assumed to be glued together at their interface, as shown in Fig. 1.

All three body components (head, thorax, and abdomen) and two wings are independently modeled, for a multibody dynamic model of the hawkmoth (Fig. 1 (a)). The head, thorax, and abdomen are connected to each other via a fixed joint, which allows no translation or rotation. The two wings are connected to the thorax via a 3-DOF revolute joint, for the wing kinematics. The body and wing morphological data are referred from measurement data [13, 14], and these are tabulated in Tables 1 and 2.

The parameters in Table 1 denote: $m_{w}$ is wing mass; $R$ is span; $\bar{c}$ is mean chord; $S$ is area; $t$ is wing thickness; and $r_{2}$ is radius of second moment of area. The mass fraction of

Table 1. Wing morphological data

\begin{tabular}{ccccccc}
\hline Parameter & $m_{w}$ & $R$ & $\bar{c}$ & $S$ & $t$ & $r_{2} / R$ \\
\hline Value & $48.33 \mathrm{mg}$ & $48.30 \mathrm{~mm}$ & $18.09 \mathrm{~mm}$ & $883.75 \mathrm{~mm}^{2}$ & $3.67 \mathrm{E}-2 \mathrm{~mm}$ & 0.51 \\
\hline
\end{tabular}

Table 2. Body morphological data

\begin{tabular}{cccccc}
\hline Parameter & $m_{\text {total }}$ & $L$ & $m_{\text {head }}$ & $m_{\text {thorax }}$ & $m_{\text {abdomen }}$ \\
\hline Value & $1456.33 \mathrm{mg}$ & $40.16 \mathrm{~mm}$ & $34.01 \mathrm{mg}$ & $481.98 \mathrm{mg}$ & $843.67 \mathrm{mg}$ \\
\hline
\end{tabular}

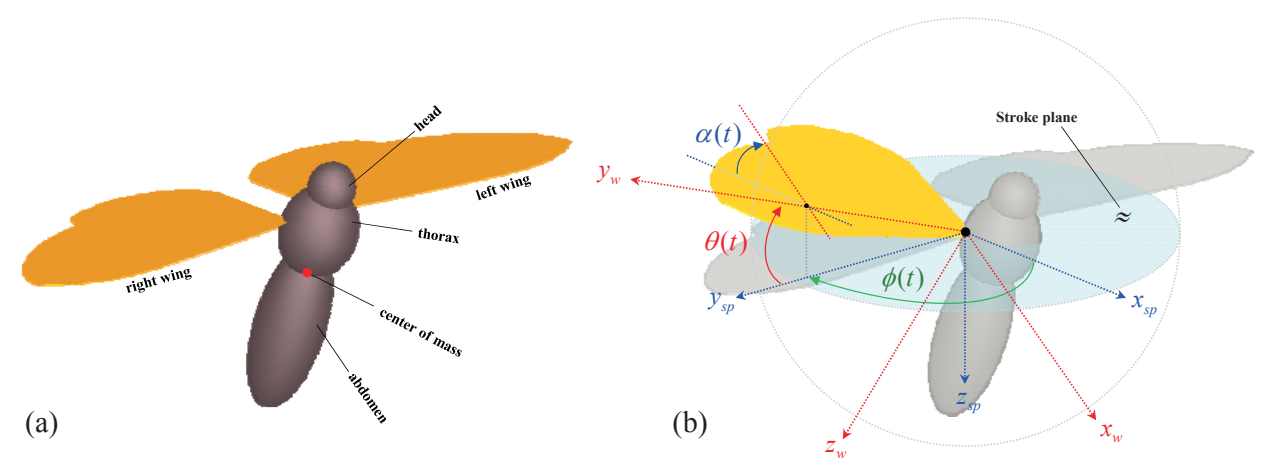

Fig. 1. (a) Multibody dynamics model of the hawkmoth, where each body component (head, thorax, and abdomen) is modeled as an ellipsoid of revolution, (b) Definition of the wing kinematics: $\phi(\mathrm{t})$, positional angle; $\alpha(\mathrm{t})$, feathering angle; and $\theta(\mathrm{t})$, deviation angle. 
the wings is about six percent of the whole body weight. The parameters in Table 2 denote: $m_{\text {total }}$ is total mass; $L$ is body length (anterior tip to posterior tip); and $m_{\text {head,thorax,abdomen }}$ is mass of each component. The location of the center of mass and the wing-base pivot are matched with the measurement data. Each component is modeled as an ellipsoid of revolution.

Four coordinate systems are required to describe the dynamics of the hawkmoth model: 1) wing-fixed $\left.\left[x_{w} y_{w} z_{w}\right], 2\right)$ stroke-plane $\left.\left[x_{s p} y_{s p} z_{s p}\right], 3\right)$ body-fixed $\left[x_{b} y_{b} z_{b}\right]$, and 4) inertial $\left[X_{G} Y_{G} Z_{G}\right]$ coordinate systems. These coordinate systems are depicted in Fig. 2. The inertial and body-fixed coordinate systems describe local/global six-degree-of-freedom (6DOF) motions; the stroke-plane and wing coordinate systems are required to compute blade element theory based aerodynamic model for the flapping wings.

To establish a multibody dynamic model of the hawkmoth, as shown in Fig. 1 (a), we used a multibody dynamics code (MSC.Adams, MSC Software Corp.). This code generates and integrates a set of nonlinear Differential and Algebraic Equations (known as a DAE system), which are based on a user-given multibody configuration (as in Fig. 1 (a)). A generic form of this DAE system is:

$$
\begin{aligned}
& M \ddot{q}+\eta_{q}^{T} \lambda-A^{T} F(q, \dot{q})=0 \\
& \eta(q, t)=0
\end{aligned}
$$

where, $M$ is the mass matrix of the system, $q$ is the set of coordinates used to represent displacements, $\eta$ is the set of configuration and applied motion constraints (kinematic constraint equations), $\lambda$ is the Lagrange multipliers for handling multiple constraints, $F$ is the set of applied forces and gyroscopic terms of the inertia forces, $A^{T}$ is the matrix that projects the applied forces in the direction $q$, and $\eta_{q}$ is the gradient of the constraints at any given state, which can be thought of as the normal to the constraint surface in the configuration space. In our hawkmoth model, there are five rigid bodies, and four kinematic constraints (two are fixed joints, and the other two are 3-DOF revolute joints).

For the forcing terms to the system, here the aerodynamics on the flapping wings are independently coded with FORTRAN, and appended as a user-written subroutine to the multibody dynamics solver. The aerodynamic model is
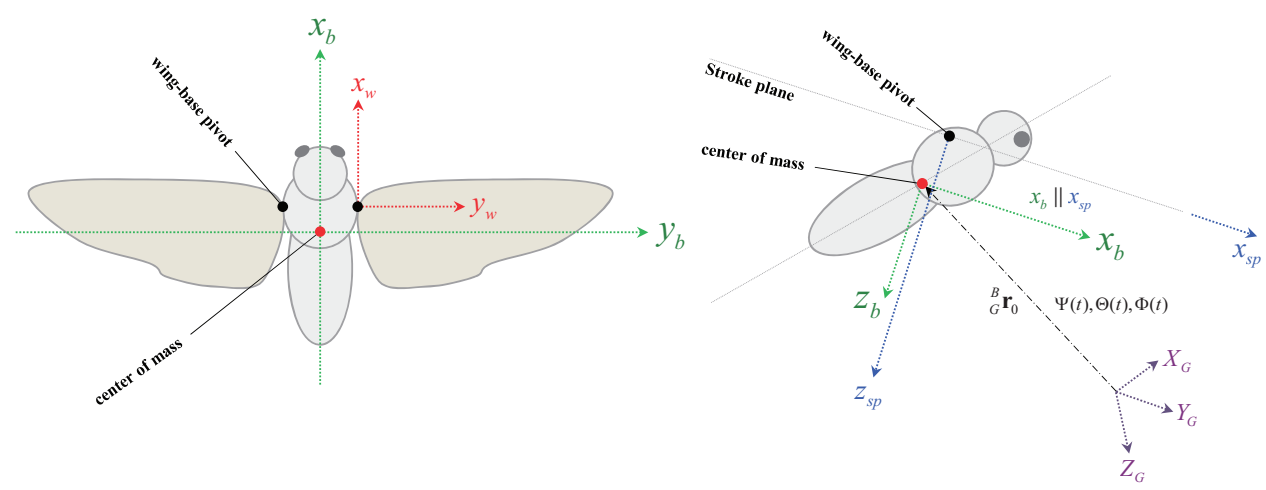

Fig. 2. Definition of the coordinate systems: wing-fixed frame $\left[x_{w} y_{w} z_{w}\right]$ is attached to the wing-base pivot, and moves in accordance with the wing motion; stroke-plane frame $\left[x_{s p} y_{s p} Z_{s p}\right]$ has its origin at the wing-base pivot, and attached to the thorax, and each wing has its own strokeplane; body-fixed frame $\left[x_{b} y_{b} z_{b}\right]$ is attached to the center of mass, and defined parallel to the stroke-plane frame.

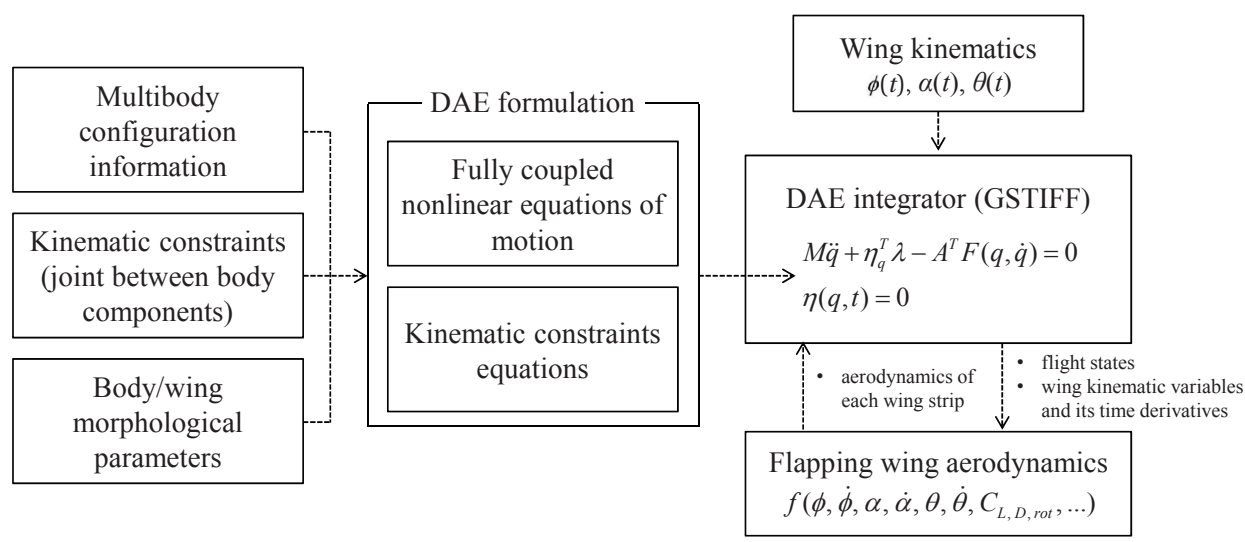

Fig. 3. A block diagram showing the modules used in multibody dynamic modeling and simulation of the hawkmoth multibody model. 
explained in section 2.2.

A block diagram, showing the modules used in multibody dynamic modeling and simulation of the hawkmoth, is given in Fig. 3.

\subsection{Aerodynamics for the flapping wings}

The aerodynamic model used in this study is based on a blade element approach, which is widely used by various research groups $[8,15,16]$. The blade element approach assumes a wing to be divided into many chord-wise aerodynamic strips. Then the aerodynamics generated from each strip are integrated along the span-wise direction, to calculate the whole wing's aerodynamic characteristics. Interactions between the wing and wake, or wing and body, are not considered in this aerodynamic model.

In this study, a total of five strips for each wing are used for the application of the blade element approach $(i=1, \ldots, 5$, starting from the wing root). The motion of each aerodynamic strip is governed by the input from the given wing kinematics (defined in section 2.3). These motions generate consequent changes in the angle of attack and incident flow velocity, which affect the aerodynamics produced by the wings, and hence alter the 6-DOF forces and moments at the center of mass of the hawkmoth model.

Eqn. 2 shows the components of an instantaneous aerodynamics, developed from a flapping motion of the wing [1].

$$
d F_{\text {instantaneous }}=d F_{\text {added-mass }}+d F_{\text {translation }}+d F_{\text {rotation }}+d F_{\text {wake-capture }}
$$

Among the four major aerodynamic components, the aerodynamics generated from a translational and a rotational motion of the wing is modeled for this study. The translational component is a quasi-steady term, which is a function of the effective angle of attack of the wing strip $\left(\alpha_{i}\right)$, and corresponding incident flow velocity $\left(V_{i}\right)$. The rotational component is an unsteady effect, generated from a rapid wing rotation that occurs at each stroke reversal, and is a function of the angular velocity of the rapid rotation, incident flow velocity, and location of the rotation axis. Eqns. 3 to 7 show each aerodynamic component's mathematical model:

$$
\begin{aligned}
& d F_{\text {lift, translation }, i}\left(V_{i}, \alpha_{i}\right)=\frac{1}{2} \rho V_{i}^{2} d S_{i} C_{L} \\
& d F_{\text {drag, translation }, i}\left(V_{i}, \alpha_{i}\right)=\frac{1}{2} \rho V_{i}^{2} d S_{i} C_{D} \\
& d F_{\text {rotation }, i}\left(\hat{x}_{0}, V_{i}, \dot{\alpha}_{i}\right)=\rho V_{i} C_{\text {rot }} \dot{\alpha}_{i} \bar{c}_{i} d S_{i} \\
& V_{i}=\left[\left(y_{w, i} \dot{\phi}(t)\right)^{2}+\left(y_{w, i} \dot{\theta}(t)\right)^{2}\right]^{1 / 2} \\
& \alpha_{i}=\alpha(t)+\tan ^{-1}\left(\frac{y_{w, i} \dot{\theta}(t)}{y_{w, i} \dot{\phi}(t)}\right)
\end{aligned}
$$

where, $\rho$ is the density of air $\left(1.225 \mathrm{~kg} / \mathrm{m}^{3}\right), d S_{i}$ is the area of each aerodynamic strip, $\bar{c}$ is the mean chord of each aerodynamic strip, and $y_{w, i}$ is the location of the aerodynamic strip $i$ from the wing-base pivot along the wing-fixed $y_{w}$-axis; $\phi(\mathrm{t}), \alpha(\mathrm{t})$, and $\theta(\mathrm{t})$ are wing kinematics variables defined in the next section 2.3.

$$
\begin{aligned}
& C_{L, t}\left(\alpha_{i}\right)=15.84 \sin \left(0.04167 \alpha_{i}+0.0262\right)+14.74 \sin \left(0.04238 \alpha_{i}-3.109\right)+0.0654 \\
& C_{D, t}\left(\alpha_{i}\right)=22.55 \sin \left(0.02481 \alpha_{i}+0.595\right)+21.87 \sin \left(0.02607 \alpha_{i}+3.761\right)+0.0983 \\
& C_{r o t, \exp }=f\left(\hat{\omega}_{i}, \hat{x}_{0, i}\right)
\end{aligned}
$$

Here, all the aerodynamic coefficients $\left(C_{L}, C_{D}\right.$, and $\left.C_{\text {rot }}\right)$ are extracted from previous experimental studies $[1,17]$. The lift and drag coefficients (Eqn. 8) for the translational component are based on experimental data obtained by Usherwood et al. [17]. They conducted a vertical/horizontal force measurement experiment with a scaled hawkmoth wing, which was rotating with an angular velocity that corresponds to $R e=8071$. We curve fitted the experimental data, to obtain the lift and drag coefficients in Eqn. 8. This is a function of the effective angle of attack $\left(\alpha_{i}\right)$, where subscript $i$ denotes the wing strip number. The unsteady rotational force coefficient (Eqn. 9) is modeled from an experimental study of Sane et al. [1]. It is a tabular form of coefficients due to the nonlinearity, and is a function of the nondimensional angular velocity of the strip $\left(\hat{\omega}_{i}=\dot{\alpha}_{i} \bar{c}_{i} / V_{i}\right)$, and the location of the nondimensional axis of rotation $\left(\hat{x}_{0, i}=x / \bar{c}_{i}\right.$, where $x$ is the length from leading edge to the feathering axis, $y_{w}$ ) of the strip. The coefficients can be found in Figure 2 of Sane et al. [1].

\subsection{Definition of wing kinematics control input vari- ables}

The motion of the insect wing can be defined with three independent angular degrees of freedom at the wing-base pivot: 1) $\phi(\mathrm{t})$, stroke positional angle, 2) $\alpha(\mathrm{t})$, feathering angle, and 3) $\theta(\mathrm{t})$, deviation angle. The stroke positional angle governs the back and forth motion of the wing; the feathering angle governs the geometrical angle of attack of the wing surface; and the deviation angle governs the up and down motion of the wing with respect to the stroke plane (see Fig. 1 (b) for a graphical representation).

Based on this wing kinematics definition, the motion of each rotational degree of freedom is defined with a sinusoidal function, to reproduce the periodic wing beat motion of the insect flight. Eqns. 10 to 12 show the wing kinematics function for each rotational degree of freedom:

$$
\begin{aligned}
& \phi(t)=\phi_{\text {amp }} \sin (2 \pi f t)+\phi_{0} \\
& \alpha(t)=\frac{\alpha_{\text {amp }}}{\tanh \left(C_{\alpha}\right)} \tanh \left(C_{\alpha} \sin \left(2 \pi f t-\frac{\pi}{2}\right)\right)+\alpha_{0}
\end{aligned}
$$




$$
\begin{aligned}
& \theta(t)=\theta_{\text {amp }} \cos (2 \pi(2 f) t)+\theta_{0} \\
& \Delta X=X_{\text {right }}-X_{\text {left }}, \quad X=[\phi, \alpha, \theta]
\end{aligned}
$$

where, $f=26 \mathrm{~Hz}$ for all the kinematic variables, which is the typical flapping frequency of the hawkmoth flight [21], and $C_{\alpha}=4.5$, which is a coefficient for adjusting the interval of the stroke reversal.

Each kinematics function has two variables, which are the amplitude and bias; and the variation of these two variables is used to evaluate the control effectiveness of the hawkmoth model. All amplitude control variables have nominal values of: $\phi_{\text {amp, nominal }}=55^{\circ}, \alpha_{\text {amp, nominal }}=45^{\circ}, \theta_{\text {amp, nominal }}=0^{\circ}$, and all bias control variables have zero nominal values (Table 3.). For the feathering angle, $\alpha(\mathrm{t}), C_{\alpha}$ is a coefficient for tuning the shape of the function: as $C_{\alpha}$ goes to infinity, the function becomes a square wave function, and as $C_{\alpha}$ goes to zero, the function becomes a sine wave [18]. Here, a square wave indicates that the stroke reversal takes place within an infinitesimal period of time, whereas a sine wave indicates a gradual reversal of the wingbeat strokes. In this study, $C_{\alpha}$ is set to 4.5 , to give a stroke reversal time of 0.15 , which is a value nondimensionalized by the wing beat stroke period $(T)$.

The abovementioned wing kinematics control input variables are depicted in Fig. 4. Each wing has three rotational degrees of freedom, and each rotational degree of freedom has two control variables; hence a total of 12 control input variables are defined, for both left and right wings. However, to obtain more intuitive results, control variable sets are divided into six symmetric and six asymmetric inputs. The
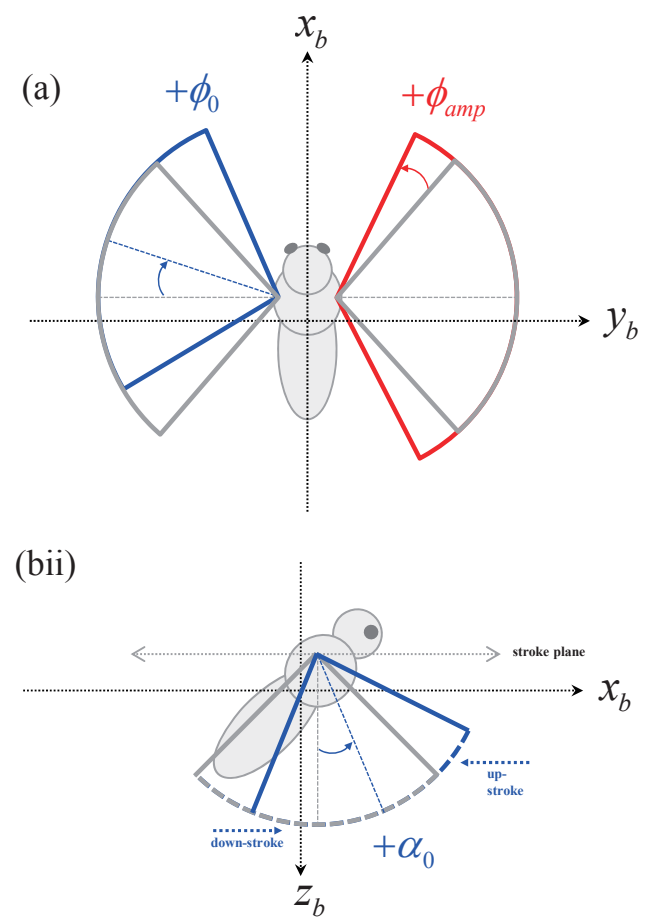

Fig. 4. Illustrations of the control inputs in each wing kinematics function symmetric inputs indicate that the control inputs to the left and right wing are identical, whereas the asymmetric inputs mean that the left and right wing have different control inputs, defined by $\Delta X$ (Eqn. 13). For example, if $\Delta \alpha_{a m p}=20^{\circ}$, the feathering angle amplitude of the right wing is larger than the left wing, by an amount of $20^{\circ}$. Considering the nominal value of the feathering angle amplitude defined in Table $3, \alpha_{\text {amp,right }}$ becomes $55^{\circ}$, and $\alpha_{\text {amp,left }}$ becomes $35^{\circ}$, which gives $20^{\circ}$ of $\Delta X$ value. The range and nominal value of each control variable are tabulated in Table 3.

\section{Results and Discussion}

The abovementioned control input variable sets for the wing kinematics are given to the hawkmoth model, to investigate their effect on the 6-DOF forces and moments. Overall, the result shows that the symmetric control

Table 3. Range and nominal values of each control input variable for the wing kinematics

\begin{tabular}{cccc}
\hline Variable & $\begin{array}{c}\text { Nominal } \\
\text { value }\end{array}$ & Range & Increment \\
\hline$\phi_{a m p}$ & $55^{\circ}$ & {$\left[30^{\circ} 80^{\circ}\right]$} & $5^{\circ}$ \\
$\alpha_{\text {amp }}$ & $45^{\circ}$ & {$\left[20^{\circ} 70^{\circ}\right]$} & $5^{\circ}$ \\
$\theta_{a m p}$ & $0^{\circ}$ & {$\left[-25^{\circ} 25^{\circ}\right]$} & $5^{\circ}$ \\
$\phi_{0}$ & $0^{\circ}$ & {$\left[-25^{\circ} 25^{\circ}\right]$} & $5^{\circ}$ \\
$\alpha_{0}$ & $0^{\circ}$ & {$\left[-25^{\circ} 25^{\circ}\right]$} & $5^{\circ}$ \\
$\theta_{0}$ & $0^{\circ}$ & {$\left[-25^{\circ} 25^{\circ}\right]$} & $5^{\circ}$ \\
\hline
\end{tabular}

\section{(bi)}

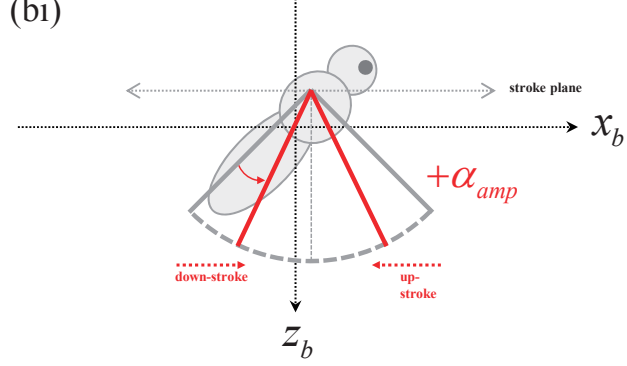

(c)

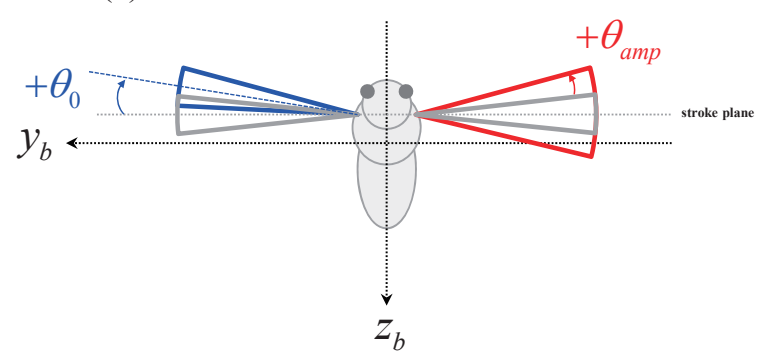


inputs affect the longitudinal forces and moments, and the asymmetric control inputs affect the lateral forces and moments. Therefore, control inputs for the longitudinal motions and lateral motions are almost decoupled.

\subsection{Longitudinal control}

Fig. 5 shows the 6-DOF forces and moments variation with respect to the symmetric wing kinematics control inputs. The ordinate indicates 6-DOF forces and moments: from above, $\mathrm{F}_{x, y, z}$ and $\mathrm{M}_{x, y, z}$. The abscissa indicates symmetric control input variables: from the left, $\phi_{a m p}, \phi_{0}, \alpha_{a m p}, \alpha_{0}, \theta_{a m p}$ and $\theta_{0}$. The shaded region specifies longitudinal forces and moments. Graphs having a thick red solid border indicate the most affected force or moment by the corresponding control input variable on the abscissa. Graphs having a thick blue dashed border indicate the second most affected force or moment by the corresponding control input variable on

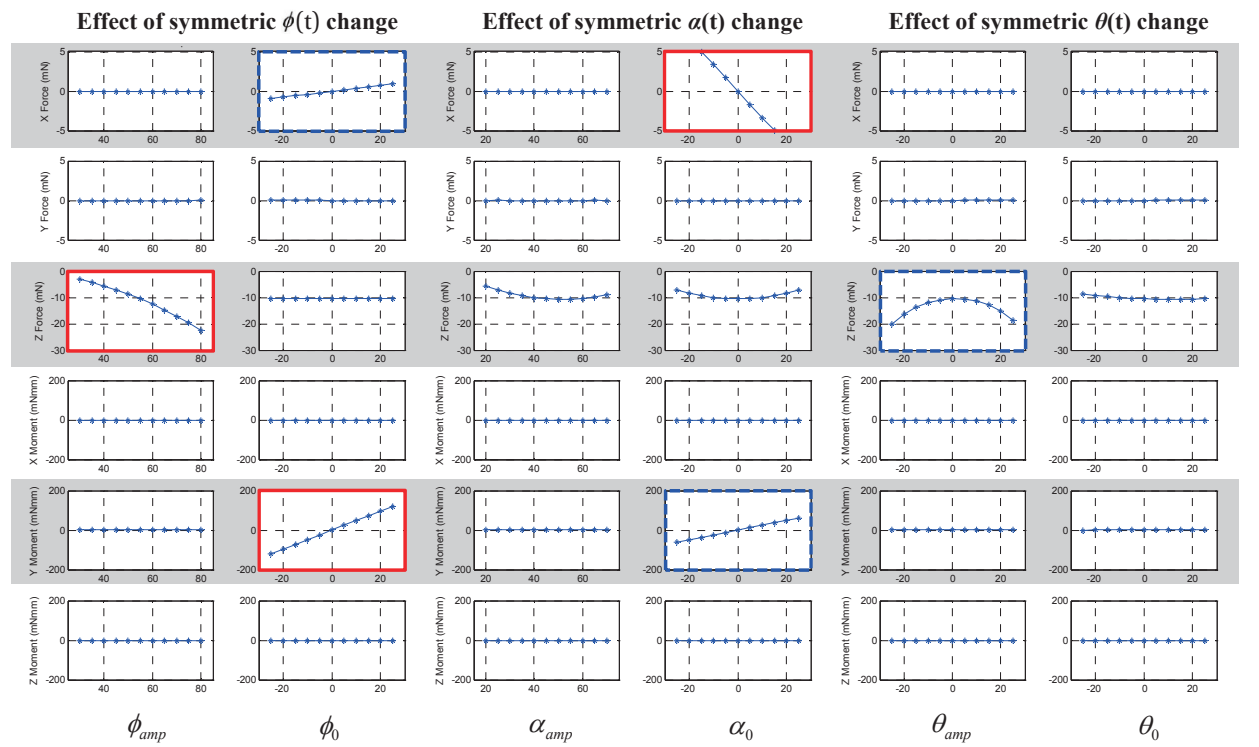

Fig. 5. Control effectiveness of symmetric control input variables to the flight dynamics (shaded region indicates longitudinal forces and moments: $F_{x}, F_{z}$ and $\left.M_{y}\right)$

Input: symmetric bias of the feathering angle $\left(\alpha_{0}\right)$

Output: forward and backward motion $\left(x_{b}\right.$ direction)

Input: symmetric amplitude of stroke positional angle $\left(\phi_{\text {amp }}\right)$

Output: descending and ascending motion $\left(z_{b}\right.$ direction)

Input: symmetric bias of the stroke positional angle $\left(\phi_{0}\right)$

Output: pitching motion $\left(y_{b}\right.$ rotational direction)
$<$ Up-stroke $>$

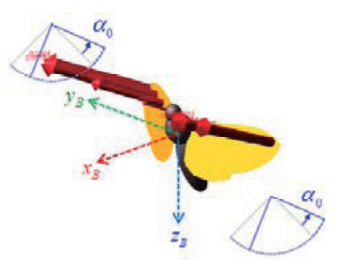

< Down-stroke >
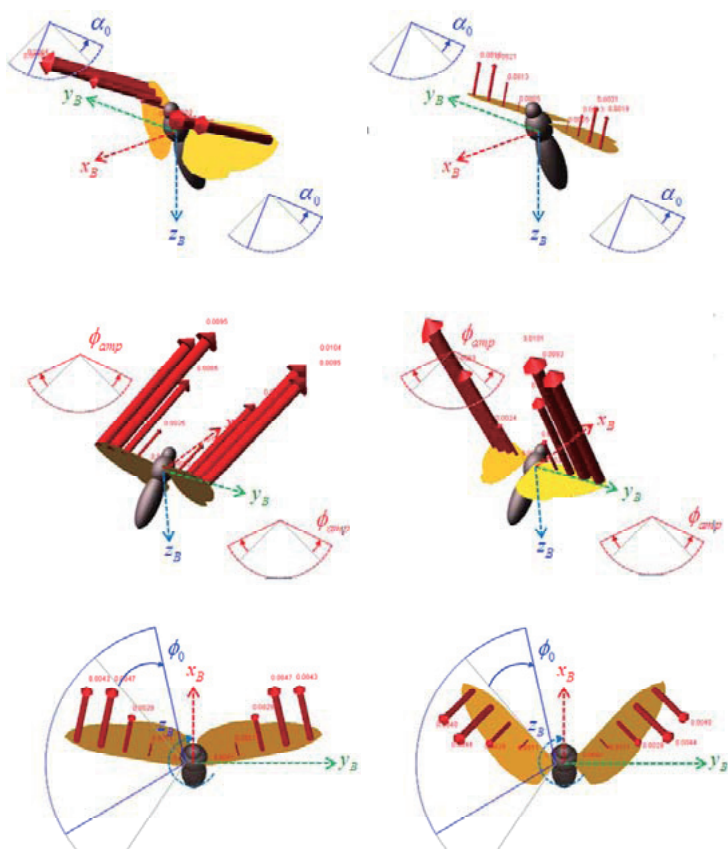

Fig. 6. Aerodynamic vectors on the wings under longitudinal control inputs 
the abscissa. As shown in Fig. 5, no symmetric control input variable affects lateral forces or moment.

The results show that the symmetric bias of the feathering angle $\left(\alpha_{0}\right)$ is needed for the control authority for the forward and backward motion ( $x_{b}$ direction). This mode of wing motion for the forward flight, termed a paddling mode, was observed from the high-speed camera measurement of a fruitfly's forward flight [3]. For the descending and ascending motion ( $z_{b}$ direction), the symmetric amplitude of stroke positional angle $\left(\phi_{a m p}\right)$ is found to be the most effective control input variable. The other control variables $\left(\alpha_{a m p}\right.$, $\alpha_{0}$, and $\theta_{\text {amp }}$, also have control authorities to this direction. However, the nonlinear variation of the $F_{z}$ force component with respect to the changes in $\alpha_{a m p}, \alpha_{0}$, and $\theta_{a m p}$, makes it ineffective to be used as a control input variable. For the pitching motion ( $y_{b}$ rotational direction), the symmetric bias

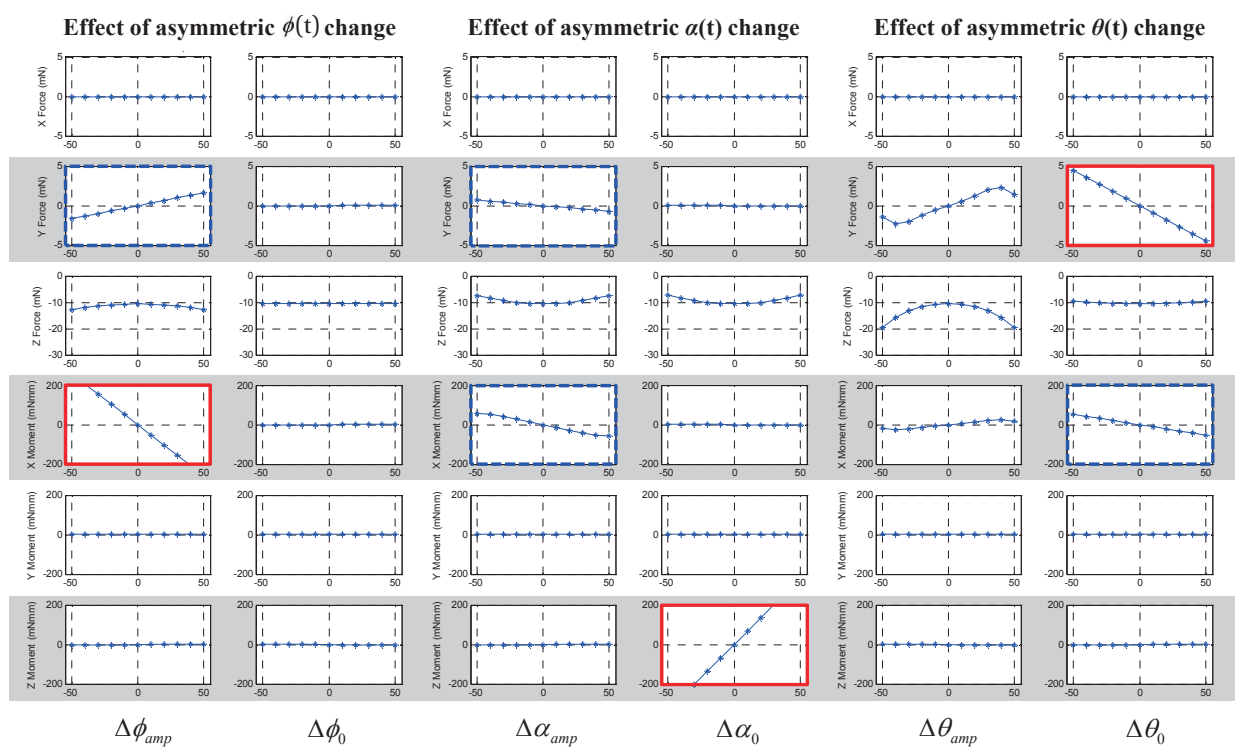

Fig. 7. Control effectiveness of asymmetric control input variables to the flight dynamics (shaded region indicates lateral forces and moments: $F_{y}$ $M_{x}$ and $\left.M_{z}\right)$

Input: asymmetric bias of the deviation angle $\left(\Delta \theta_{0}\right)$

Output: side-translation motion $\left(y_{b}\right.$ direction)

Input: asymmetric amplitude of the stroke positional angle $\left(\Delta \phi_{a m p}\right)$

Output: roll motion $\left(x_{b}\right.$ rotational direction)

Input: asymmetric bias of the feathering angle $\left(\Delta \alpha_{0}\right)$

Output: yaw motion $\left(z_{b}\right.$ rotational direction)
$<$ Up-stroke >
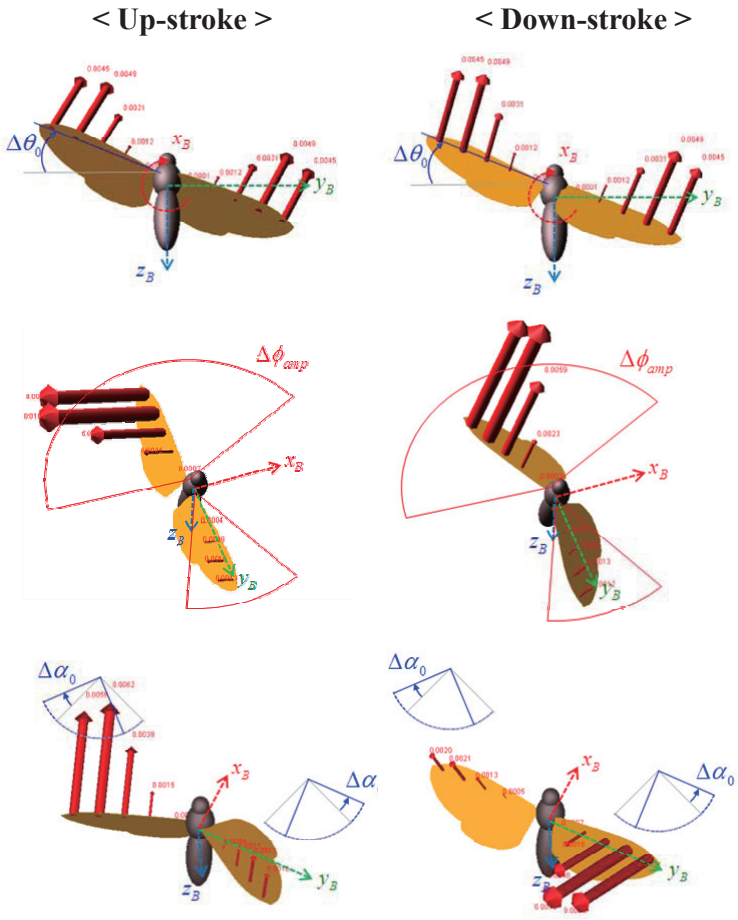

Fig. 8. Aerodynamic vectors on the wings under lateral control inputs 
of the stroke positional angle $\left(\phi_{0}\right)$ is needed for the control authority. A shift in the mean value of the stroke positional angle results in a shift of the aerodynamic center, during a wing beat cycle, to forward or backward with respect to the center of mass; therefore a pitching moment is generated. Fig. 6 illustrates the aerodynamic vectors on the wings, under the given wing kinematics control inputs for the longitudinal control forces and moments.

\subsection{Lateral control}

Fig. 7 shows the 6-DOF forces and moments variation with respect to the asymmetric wing kinematics control inputs. The asymmetric control inputs are defined in section 2.3. The ordinate indicates 6 -DOF forces and moments: from above, $F_{x, y, z}$ and $M_{x, y, z}$. The abscissa indicates asymmetric control input variables: from the left, $\Delta \phi_{a m p}, \Delta \phi_{0}, \Delta \alpha_{a m p}, \Delta \alpha_{0}$, $\Delta \theta_{\text {amp }}$, and $\Delta \theta_{0}$. The shaded region specifies lateral forces and moments. The convention for the thick solid/dashed border is the same as for the longitudinal control effectiveness.

The results show that the asymmetric bias of the deviation angle $\left(\Delta \theta_{0}\right)$ is the most effective control input for the control authority to the side-translation motion ( $y_{b}$ direction). This asymmetric bias of the deviation angle directly tilts the aerodynamic force vectors to one direction, by tilting the whole stroke plane. For the roll motion $\left(x_{b}\right.$ rotational direction), the asymmetric amplitude of the stroke positional angle $\left(\Delta \phi_{\text {amp }}\right)$ is needed to generate roll moment. The asymmetric bias of the feathering angle $\left(\Delta \alpha_{0}\right)$ is found to be the most effective, and also the only control input for the control authority to the yaw motion ( $z_{b}$ rotational direction). Also, this strategy of yaw control was demonstrated by the Nano hummingbird platform, developed by AeroVironment Inc. [2]. Fig. 8 illustrates the aerodynamic vectors on the wings, under the given wing kinematics control inputs for the lateral control forces and moments.

\subsection{Coupling effect of control inputs}

As shown in the Figs. 5 and 7, some control input variables affect not only a single directional force or moment (graphs with thick solid border), but they also simultaneously affect two or three forces or moments (graphs with thick dashed border). This means that there is a coupling effect between DOFs with respect to a single control input variable. However, there is not much coupling between longitudinal and lateral DOFs, except only for the longitudinal up/down motion with the lateral dynamics.

In the longitudinal DOFs ( $x_{b}$ translation, $z_{b}$ translation, and $y_{b}$ rotation), $F_{x}$ and $M_{y}$ always couple together: forward/ backward force and pitching moment. In Fig. 5, both the symmetric bias of the stroke positional angle $\left(\phi_{0}\right)$ and the symmetric bias of the feathering angle $\left(\alpha_{0}\right)$ have an effect on the $F_{x}$ and $M_{y}$, with different direction. An increase of the symmetric bias of the stroke positional angle $\left(\phi_{0}\right)$ induces increases both in $F_{x}$ and $M_{y}$ (in-phase), which means that the hawkmoth pitches up (down), while going forward (backward) at the same time. This mode of motion is known to be highly unstable $[5,19]$, hence further attitude stabilization control is necessary for using this control strategy. On the other hand, increase of the symmetric bias of the feathering angle $\left(\alpha_{0}\right)$ induces a decrease in $F_{x}$, and an increase in $M_{y}$ (out-of-phase). This means that the hawkmoth pitches down (up), while going forward (backward), and this mode of motion is known to be stable $[5,19]$. By taking advantage of this coupling effect, it seems that the symmetric bias of the feathering angle $\left(\alpha_{0}\right)$ is appropriate for the attitude control to the directions of forward/backward and pitching motions.

In the lateral DOFs ( $y_{b}$ translation, $x_{b}$ rotation, and $z_{b}$ rotation), $F_{y}$ and $M_{x}$ always couple together: side-translation force and roll moment. Fig. 7 shows that three control inputs have an effect on $F_{y}$ and $M_{x}$, which are the asymmetric amplitude of the stroke positional angle $\left(\Delta \phi_{a m p}\right)$, the asymmetric amplitude of the feathering angle $\left(\Delta \alpha_{\text {amp }}\right)$, and the asymmetric bias of the deviation angle $\left(\Delta \theta_{0}\right)$. Among these three control inputs, an increase of $\Delta \alpha_{a m p}$ and $\Delta \theta_{0}$ cause an increase both in $F_{y}$ and $M_{x}$ (in-phase). This portrays a situation where the hawkmoth goes right (left), while rolling right (left), and this mode of motion is known to be unstable [20]. Therefore, initiating this mode of motion with a certain control input will make the insect flight diverge, thus further compensation is needed for stabilization. However, an increase of $\Delta \phi_{a m p}$ induces an increase in $F_{y}$ and a decrease in $M_{x}$ (out-of-phase), which renders a situation where the hawkmoth translates right (left) while rolling left (right). This mode of motion is known to be stable [20]. Therefore, for the control of side-translation and roll motion, the asymmetric amplitude of the stroke positional angle $\left(\Delta \phi_{a m p}\right)$ seems to be a proper choice.

In terms of a cross-coupling between motion planes (longitudinal and lateral), there is a longitudinal force component, $F_{z}$, which is affected by the asymmetric lateral control inputs. It is shown in Fig. 7, and most of the asymmetric control inputs have a slight effect on the overall lift production of the wing. This seems to be the effect of the inclined aerodynamic vector from the wing compared to a nominal condition, due to the altered wing kinematics. This slight increase or decrease in the lift force must be taken into account, when designing a robust flight controller.

\section{Conclusion}

A control effectiveness analysis is conducted using a multibody dynamics model of the hawkmoth Manduca sexta. From the analyses, the sensitivities of defined control input variables to the 6-DOF flight forces and moment are investigated, and their coupling effects are also examined. 
For the longitudinal flight: 1) the symmetric bias of the feathering angle $\left(\alpha_{0}\right)$ is effective for the forward/backward and pitching motion control, and 2) the symmetric amplitude of the stroke positional angle $\left(\phi_{\text {amp }}\right)$ is effective for the descending and ascending motion.

For the lateral flight: 1) the asymmetric bias of the feathering angle $\left(\Delta \alpha_{0}\right)$ is effective for the yaw motion control, and 2) the asymmetric amplitude of the stroke positional angle $\left(\Delta \phi_{a m p}\right)$ is effective for the control of side-translation and roll motion.

From the coupling effect analysis, reduction in the number of necessary control input variables is achieved. Among all twelve possible combinations of control variables for three rotational degrees of freedom of the wing, only four of them $\left(\alpha_{0}, \Delta \alpha_{0}, \phi_{a m p}\right.$, and $\left.\Delta \phi_{\text {amp }}\right)$ are found to be sufficient for the control of 6-DOF forces and moments. From an engineering point of view, reduction of the number of required control inputs directly translates to a simple mechanism with less weight, which can lead us to alleviate the criteria for actuators, sensors, and computational burdens. We hope that this study can help in the designing of efficient flight controllers for flapping-wing MAVs.

\section{Acknowledgement}

The Authors gratefully acknowledge the support from the UTRC (Unmanned technology Research Center) at KAIST, originally funded by DAPA and ADD. The first author thanks the project of Global Ph.D. Fellowship, which the National Research Foundation of Korea has conducted from 2011.

\section{References}

[1] Sane, S. P., and Dickinson, M. H., "The aerodynamic effects of wing rotation and a revised quasi-steady model for flapping flight", Journal of Experimental Biology, Vol. 205, No. 8, 2002, pp. 1087-1096.

[2] Keennon, M., Klingebiel, K., Won, H., and Andriukov, A., "Development of the Nano Hummingbird: A Tailless Flapping Wing Micro Air Vehicle", Proceedings of the 50th AIAA Aerospace Sciences Meeting including the New Horizons Forum and Aerospace Exposition, Nashville, TN, 2012, Article number: AIAA 2012-0588.

[3] Ristroph, L., Bergou, A., Guckenheimer, J., Wang, Z., and Cohen, I., "Paddling Mode of Forward Flight in Insects", Physical Review Letters, Vol. 106, No. 1, 2011, pp. 178103-1 178103-4.

[4] Walker, S. M., Thomas, A. L. R., and Taylor, G. K., "Deformable wing kinematics in free-flying hoverflies", Journal of the Royal Society Interface, Vol. 7, No. 42, 2010, pp.
131-142.

[5] Cheng, B., Deng, X., and Hedrick, T., "The mechanics and control of pitching manoeuvres in a freely flying Hawkmoth (Manduca Sexta)", Journal of Experimental Biology, Vol. 214, No. 1, 2011, pp. 4092-4106.

[6] Zhang, Y., and Sun, M., "Control for small-speed lateral flight in a model insect", Bioinspiration and Biomimetics, Vol. 6, No. 3, 2011, pp. 036003-1 - 036003-9.

[7] Pérez-Arancibia, N. O., Ma, K. Y., Galloway, K. C., Greenberg, J. D., and Wood, R. J., "First controlled vertical flight of a biologically inspired microrobot", Bioinspiration and Biomimetics, Vol. 6, No. 3, 2011, pp. 036009-1 - 03600911.

[8] Paranjape, A., Dorothy, M. R., Chung, S.-J., and Lee, K. D., "A Flight Mechanics-Centric Review of Bird-Scale Flapping Flight", International Journal of Aeronautical and Space Sciences, Vol. 13, No. 3, 2012, pp. 267-281.

[9] Orlowski, C. T. and Girard, A. R., "Modeling and Simulation of Nonlinear Dynamics of Flapping Wing Micro Air Vehicles", AIAA J., Vol. 49, No. 5, 2011, pp. 969-981

[10] Lee, J.-S., Kim, J.-K., Kim, D.-K., and Han, J.-H., "Longitudinal Flight Dynamics of Bioinspired Ornithopter Considering Fluid-Structure Interaction", Journal of Guidance, Control and Dynamics, Vol. 34, No. 3, 2011, pp. 667-677.

[11] Kim, J.-K., Lee, J.-S., and Han, J.-H., "Passive Longitudinal Stability in Ornithopter Flight", Journal of Guidance, Control and Dynamics, Vol. 35, No. 2, 2012, pp. 669-673.

[12] Jantzen, B., and Eisner, T., "Hindwings are unnecessary for flight but essential for execution of normal evasive flight in Lepidoptera", Proceedings of the National Academy of Sciences, Vol. 105, No. 43, 2008, pp. 16636-16640.

[13] Ellington, C. P., "The aerodynamics of hovering insect flight: II. Morphological parameters", Philos. Trans. R. Soc. B-Biol. Sci. Vol. 305, No. 1122, 1984, pp. 17-40.

[14] O'Hara, R. P., and Palazotto, A. N., “The morphological characterization of the forewing of the Manduca sexta species for the application of biomimetic flapping wing micro air vehicles", Bioinspiration and Biomimetics, Vol. 7, No. 4, 2012, pp. 046011-1 - 046011-13.

[15] Truong, Q. T., Nguyen, Q. V., Truong, V. T., Park, H.-C., Byun, D.-Y., and Goo, N.-S., "A modified blade element theory for estimation of forces generated by a beetle-mimicking flapping wing system", Bioinspiration and Biomimetics, Vol. 6, No. 3, pp. 036008-1 - 036008-11.

[16] Doman, D. B., Oppenheimer, M. W., and Sigthorsson, D. O., "Wingbeat Shape Modulation for Flapping-Wing Micro-Air-Vehicle Control During Hover", Journal of Guidance, Control and Dynamics, Vol. 33, No. 3, 2010, pp. 
724-739.

[17] Usherwood, J. R., and Ellington, C. P., “The aerodynamics of revolving wings, I. Model Hawkmoth wings" , Journal of Experimental Biology, Vol. 205, No. 11,2001, pp. 1547-1564.

[18] Berman, G. J., and Wang, Z., "Energy-minimizing kinematics in hovering insect flight", Journal of Fluid Mechanics, Vol. 582, No. 1, 2007, pp. 153-168.

[19] Sun, M., Wang, J., and Xiong, Y., "Dynamic flight stability of hovering insects", Acta Mech. Sin., Vol. 23, No. 3, 2007, pp. 231-246.

[20] Zhang, Y.-L., and Sun, M., "Dynamic flight stability of a hovering model insect: lateral motion", Acta Mech. Sin., Vol. 26, No. 2, 2010, pp. 175-190.

[21] Willmott, A. P., and Ellington, C. P., "The mechanics of flight in the hawkmoth Manduca sexta. I. Kinematics of hovering and forward flight", Journal of Experimental Biology, Vol. 200, No. 21, 1997, pp. 2705-2722 